\title{
The effect of serial data collection on the accuracy of Electrical Impedance Tomography images
}

\author{
Rebecca Yerworth ${ }^{1}$ and Richard Bayford ${ }^{2}$ \\ 1. Sir Harry Smith Community College, Whittlesey, PE7 1Xb \\ 2.Middlesex University, Department of Natural Science, London, NW 4BT \\ E-mail: rebecca.yerworth@googlemail.com
}

\begin{abstract}
There has been a surge of interest in using Electrical Impedance Tomography (EIT) for monitoring regional lung ventilation however EIT is an ill-conditioned problem, and errors/noise in the boundary voltages can have an undesirable effect on the quality of the final image. Most EIT systems in clinical usage use serial data collection hence data used to create a single image will have been collected at different times. This paper presents a study of the resulting image distortion, and proposes a method for correcting this lag in situations where the frame rate is insufficient to prevent significant image degradation. Significant correlation between the standard deviation of the time dependent reciprocity error and time delay $\mathrm{dL}_{\mathrm{e}}$ between the reciprocal electrode combinations was found for both adult and neonate data. This was reduced when the data was corrected for $\mathrm{dL}_{\mathrm{e}}$. Original and corrected data was reconstructed with the GREIT algorithm and visible differences were found for the neonate data.
\end{abstract}

Ideally EIT systems should be run at a frame rate of at least 50 times the frequency of the dominant and interesting physiological signals. Where this is not practical, the intra-frame system timings should be determined and lag corrected for.

\section{Introduction}

Electrical Impedance Tomography (EIT) measures the internal impedance of a body from a series of surface electrodes placed on the surface of the body. It is capable of providing real-time spatial information related to structural and physiological changes. EIT offers the opportunity for continuous, non-invasive, bedside monitoring of lung ventilation (Grychtol et al 2010). In recent years there has been a surge of interest in using EIT for monitoring regional lung ventilation (Adler et al 2012), with particular interest in it's application to management of acute lung injury (ALI) and acute respiratory distress syndrome (ARDS).

EIT is an ill-conditioned problem, and errors/noise in the boundary voltages can have an undesirable effect on the quality of the final image. The most successful use of EIT in lung imaging is based on reconstructed images of normalised difference changes relative to a baseline frame (e.g. tidal breathing prior to adjustment of ventilator settings), since this approach reduces the effect of systematic errors in the data (Adler et al 2012).

The first EIT images were reconstructed using back projection techniques, and a range of different algorithms have been used since. The GREIT reconstruction algorithm (Graz consensus Reconstruction algorithm for EIT) (Adler et al 2009) set a new standard for clinical EIT. Grychtol et al (2012) studied the effect of model shape mismatch on reconstruction quality, concluding that small shape mismatches were well tolerated by the GREIT algorithm, but that using a circular model had a strong detrimental effect on image quality, building on earlier work by Bayford et al (2008). Earlier, others have looked at the effect of dynamic non-idealalities in the data collection hardware (Hahn et al 2008, Hartinger et al 2007), and found that this is only significant if absolute or frequency difference imaging is to be considered. The exclusion of electrode combinations with 
excessive noise or non-physiological artefacts was considered by the UCL group (Tidswell et al 2001) and Adler (2004).

Gomez-laberge et al (2012) have published "a unified approach for EIT imaging ...in acute lung imaging", building on recently proposed methods of regional lung volume measurements. These previous papers all assumed "that a reconstruction algorithm has already computed images from the voltage measurements" (Gómez-laberge et al (2012), p840), and in most cases this was based on a backprojection algorithm available with commercial EIT systems. Gomez-Laberge et al used the newer GREIT reconstruction algorithm (Adler et al 2009), recognising that the choice of reconstruction algorithm affects the images and thus the derived parameters. They state that electrode movement was taken into account during the image reconstruction, as facilitated by the GREIT algorithm, but mention no other error correction or preprocessing of the EIT boundary voltage data.

Whilst the use of new GREIT reconstruction algorithms has improved imaged quality, and provided a unified approach to reconstructing images, there remains the implicit assumption that all data for a single image frame is collected from an effectively static measurement state. However most EIT systems in clinical usage use serial data collection methods, collecting data for each electrode combination sequentially, thus data used to create a single image will have been collected at different times. Whilst frame rates vary, the 16 electrodes Geo-MF II operating at 13 frames per second is not atypical. A healthy adult at rest typically takes 16-18 breaths per minute and has a pulse rate of 60-80beats per minute (bpm) but a neonate may have a breathing rate in excess of 60 breaths per minute, and an even faster heart rate, therefore physiological changes will occur within the time it takes to collect one frame of EIT data. Not only could this cause some blurring of the temporal information, but the effect may vary across the image leading to distortion and an unknown effect upon derived parameters. This paper presents a detailed study of this, examining the effect on the reconstructed images in a range of clinical situations, and proposing a method for correcting this lag in situations where the frame rate is insufficient to prevent significant image degradation.

\section{Methodology}

Reciprocity theory was used to produce a surrogate measure of lag induced error in the boundary voltage measurements.

Theoretically the voltage measured using electrodes $\mathrm{a} \& \mathrm{~b}$ to inject, and $\mathrm{x} \& \mathrm{y}$ to sense (voltage measurement) should be identical to that using $\mathrm{x} \& \mathrm{y}$ to inject and $\mathrm{a} \& \mathrm{~b}$ to sense.

This phenomena, known as reciprocity can be used to characterise electrode errors, of specific measurement systems (Oh et al 2007), and more generically (Hartinger et al 2009) and it is known that "reciprocity errors" affecting the quality of the reconstructed images (Yerworth \& Bayford 2011). EIT systems based on the original Sheffield Mk1 (Brown and Seagar 1987 ) collect data from both these reciprocal electrode combinations (reciprocity pairs) during each measurement frame. Reciprocity errors due to system effects are likely to very only slowly with time. However, if the measurement object varies during one frame, true physiological changes will appear as reciprocity errors. Even if the physiological changes are periodic, these 'errors' will vary with time, since the EIT system is not normally time-locked to the physiological signal and will be sampling it at differing points each frame. Thus calculating the standard deviation of the reciprocity 'error' will provide an indication of the severity of this issue. The time delay, or lag, between reciprocal electrode combinations is combination specific. The larger the lag the more opportunity there is for the physiological signal to change, i.e. one would expect a correlation between lag and the standard deviation of the reciprocity errors.

If the lags are known and constant, and the physiological signal vary smoothly with time, it is theoretically possible to correct for this lag, by transforming the data into the frequency domain, and adding a frequency dependant phase shift.

Previously collected data from the Middlesex University (MU)/Great Ormond Street Hospital $(\mathrm{GOSH})$ group were used. Data was acquired using the VIASYS healthcare EIT system (Viasys 
Heathcare, Hochberg, Germany (now Carefusion http://www.carefusion.com )) using an adjacent stimulation and measurement pattern. MatlabR2011b (www.mathworks.com) with Eidors-v3.6 (eidors3d.sourceforge.net) was used for data and image analysis and Minitab 16 (www.minitab.com) for statistical analysis.

\section{Method}

\section{Modelling}

A FEM mesh of the thorax, incorporating lungs, spine/sternum and heart compartments with realistic conductivities (Minhas \& Reddy 2005) was used to model the effect of lag on image reconstruction. Boundary voltages being calculated for the four scenarios using Eidors-V3.6 to solve the forward problem (Table 1). In each case the lung conductivity was uniformly changed, all other conductivity's remaining fixed. In practice 208 simulations were run for "serial-collection" with the lung conductivity ramped from +0.05 to $+10 \%$, and the boundary voltage for one electrode combination extracted from each.

\begin{tabular}{|l|l|}
\hline \multicolumn{1}{|c|}{ Frame } & \multicolumn{1}{c|}{ Lung conductivity } \\
\hline baseline & $-10 \%$ \\
\hline static1 & $+0.05 \%$ \\
\hline static2 & $+10 \%$ \\
\hline serial_collection & $\begin{array}{l}\text { Varied for each boundary voltage measurement. } \\
\text { For the } \mathrm{n}^{\text {th }} \text { electrode combination: }+10 / 208 * \mathrm{n} \%\end{array}$ \\
\hline
\end{tabular}

\section{Table 1: Simulated data}

Data was normalised to 'baseline' and reconstructed using the GREIT algorithm (Adler et al 2009), using the library model adult male mesh.

\section{Data collection}

Sixteen electrodes (Philips 13953D neonatal ECG electrodes) were attached, equally spaced, around the thorax of a subject, on a plane between the apex of the axillae and the nipple line. Electrodes were numbered in a counter-clockwise manner such that electrode one was placed on the sternum, and electrode 5 on the left mid-axcillary line. A ground electrode was placed on the anterior abdominal wall. An adjacent drive and receive protocol was used.

Sets of boundary voltages were collected in 1 minute blocks at a rate of 13 frames a second, 208 electrode combinations per frame, from two subjects, one adult female and one un-sedated neonate, each subject was positioned first on their left side and then on their right. The adult was instructed to perform $30 \mathrm{~s}$ of tidal breathing followed by 3 maximal breaths, the neonate was left to spontaneous quiet breathing (being too young to comply with instructions) (Yerworth \& Bayford 2007).

\section{Reciprocity and phase correction}

For each data set the first $2^{\wedge} 7$ frames were considered, in each case this consisted of tidal breathing, the largest tidal breathing sample available, satisfying the $2^{\wedge} n$ data point requirement of Fast Fourier transforms (FFT)

Relative delay on each data point was estimated as a linear function of the data collection order, with a delay $\left(\mathrm{L}_{\mathrm{e}}\right)$ of $0 \mathrm{~ms}$ on the $1^{\text {st }}$ electrode combination and $76.6 \mathrm{~ms}$ for the $208^{\text {th }}$ electrode combination, based on the stated frame rate of $13 \mathrm{~Hz}$. The time difference $\left(\mathrm{dL}_{\mathrm{e}}\right)$ for each reciprocity pair was calculated from this.

A Fast Fourier Transform was applied to the data set then correction for lag was made by applying a frequency dependant phase adjustment [1]. 


$$
\frac{2 * p i * f * L_{e} * F}{2^{7}}
$$

where $\mathrm{F}$ is the system frame rate $(13 \mathrm{~Hz})$ and $\mathrm{f}$ is the frequency of each point in the transformed data, up to the Nyquist frequency.

This was followed by application of an inverse Fourier transform was then performed on the corrected frequency spectrum, and the real part of this formed the lag-corrected time series data.

Reciprocity errors $\left(\mathrm{R}_{\mathrm{e}}\right)$ were calculated [2] for each electrode combination (e) and it's reciprocity pair (er)

$$
R_{e}=\left|\frac{v_{e}-v_{e r}}{\left(v_{e}+v_{e r}\right) / 2}\right| * 100
$$

The standard deviation (std) of the time dependent reciprocity error was calculated and correlated with the time delay $\mathrm{dL}_{\mathrm{e}}$. ( Fig.1).

Normalised time-difference images were reconstructed, for the original (Io) and corrected (Ic) data, using the GREIT algorithm (Adler et al 2009), using the library model neonate and adult male meshes respectively for the neonate and adult data. In both cases the noise regularisation parameter was set to 0.6 (Gómez-laberge et al 2012). The dc component of the frequency spectrum was used as the reference, which was equivalent to using the mean frame.

The difference between the original and corrected images was assessed by subtracting the two image sets on a pixel by pixel basis to create a difference image set (Id). A mean difference image (mId) and one of the standard deviation of the differences (sId) were then calculated.

\section{Results}

Images of both the static and serial simulation data show two clearly defined lungs, with intensity peak at the centre of each. In both 'static1' and 'static2' the lung to the right of the image shows slightly larger changes, however in the 'serial_collection' image this is reversed (Fig.2). The $5^{\text {th }}$ image is the difference between static1 and serial_collection.

The frequencies of the main respiratory and cardiac related signals were obtained from peaks in the frequency domain spectrum (Table 2)

\begin{tabular}{|l|l|l|}
\hline & Respiratory frequency $(\mathrm{Hz})$ & Cardiac Frequency $(\mathrm{Hz})$ \\
\hline Neonate & 0.91 & 2.4 \\
\hline Adult & 0.2 & $1.4-1.7$ \\
\hline
\end{tabular}

Table 2: Main Respiratory and cardiac frequencies in the data used

Original and lag-corrected data, from the neonate, for one pair of reciprocity measurements, where the $2^{\text {nd }}$ measurement is taken $62.9 \mathrm{~ms}$ after the first, shows the temporal shifting of the signal and improved correspondence of the lag corrected data (Fig.3). For the normal electrode arrangement, changes of up to $10 \%$ of the peak-to-peak voltage can be seen, and lag correction has shifted the peak inspiration and expiration by one measurement frame for the raw data, to be in synchrony with the data for the reciprical pair.

Correlation between Standard deviation of reciprocity errors $\left(\operatorname{std}\left(\mathrm{R}_{\mathrm{e}}\right)\right)$ and the time lag between data collection for each reciprocity pair $\left(\mathrm{dL}_{\mathrm{e}}\right)$ are given for neonate, and adult data (Table2). The correlation coefficient was highest for the uncorrected neonate data and lowest for the corrected adult data. In both cases the corrected data showed less correlation than the uncorrected data. The correlations for the corrected data show a non-linear component (Fig.4)

\begin{tabular}{|l|l|l|l|l|l|}
\hline & & Uncorrected & \multicolumn{2}{|c|}{ Lag Corrected } \\
\hline & $\begin{array}{l}\text { Mean }\left(\mathrm{R}_{\mathrm{e}}\right) \\
\left(\begin{array}{l}\text { median, } \\
\text { interquartile range) }\end{array}\right.\end{array}$ & $\begin{array}{l}\text { Std }\left(\mathrm{R}_{\mathrm{e}}\right) \\
\left(\begin{array}{l}\text { median, } \\
\text { interquartile range })\end{array}\right.\end{array}$ & $\begin{array}{l}\text { Correlation } \\
\text { std }\left(\mathrm{R}_{\mathrm{e}}\right) \&\left(\mathrm{dL}_{\mathrm{e}}\right)\end{array}$ & $\begin{array}{l}\text { Std }\left(\mathrm{R}_{\mathrm{e}}\right) \\
\left(\begin{array}{l}\text { median, } \\
\text { interquartile range })\end{array}\right.\end{array}$ & $\begin{array}{l}\text { Correlation } \\
\text { std }\left(\mathrm{R}_{\mathrm{e}}\right) \&\left(\mathrm{dL}_{\mathrm{e}}\right)\end{array}$ \\
\hline Neonate & $1.2(0.4-2.7)$ & $0.32(0.17-0.47)$ & $0.701 *$ & $0.17(0.08-0.22)$ & $0.491^{*}$ \\
\hline
\end{tabular}


Table 2: Median and interquartile range of Mean and Std of reciprocity error $\left(\mathrm{R}_{\mathrm{e}}\right)$ for the 208 electrode combinations, and the Correlation coefficient of std reciprocity and the time lag ( $\left.\mathrm{dL}_{\mathrm{e}}\right)$ between data collection for each reciprocity pair $(*$ denoted $p<0.001)$

Reconstructed images of the neonate data are shown in Fig.5, and for the adult data in Fig.7.

The peak of the mean difference image (Fig.6a) is $-4 \%$ of the maximum intensity of the original image, for the neonate and $-0.4 \%$ for the Adult. The standard deviation of the difference images ( Fig.6b), ranges from 0 to $26 \%$ of the maximum intensity of the original image for the Neonate, and 0 to $8 \%$ for the Adult.

\section{Discussion}

The images generated for the simulated data (Fig.2) demonstrate that serial data collection affects the images in a non-uniform manner. In this case the lung conductivity was increasing (and impedance decreasing) over time, from that in static1 to that in static2. The serial-collection image is not, however a simple average of the start and end conductivities, instead the right had lung resembles the initial conductivity (static1) whilst the left hand lung is much closer to the final conductivity. This makes sense, given that, for this data collection protocol, the drive combination rotates clockwise round the body starting at the sternum. So we have a problem, at least in theory, but how significant is it in clinical practice?

A significant correlation was found between the standard deviation of the reciprocity errors and $\mathrm{dL}_{\mathrm{e}}$ using lung ventilation data of neonate and adults (Table2). In both cases the standard deviation of the reciprocity errors was reduced when the phase correction was applied, to approximately the level achievable in a saline filled tank. It was predicted that the effect of $\mathrm{dL}_{\mathrm{e}}$ would be highest in the data set with the fastest changing physiological signals, i.e. the neonate data, and indeed higher standard deviation of the reciprocity errors was seen in the neonate data set than in the adult.

Looking at the original and $\mathrm{dL}_{\mathrm{e}}$ corrected data for a representative reciprocity pair (Fig.3), it can be seen that one of the uncorrected data traces lags behind the other, which is not the case for the two corrected traces. Combined with the reduction in standard deviation of the reciprocity error, this indicates that the phase correction counteracted some of the errors caused by the serial nature of the data collection. The question however, is does this make any difference to the reconstructed images, and parameters derived from the images?

Visible differences can be seen between the original and uncorrected neonate images (Fig.5a\&b), though the adult images show almost no differences. This provides reassurance that frame rates as slow as $13 \mathrm{~Hz}$ are likely to be sufficient for monitoring respiration in unventilated adults, but gives cause for concern with respect to higher frequency events, such as neonate respiration. It is perhaps surprising that the adult images showed so little difference due to the lag correction, as the correlation of the standard deviation of the reciprocity error and $\mathrm{dL}_{\mathrm{e}}$ was reduced by approximately the same amount as for the neonate data. A possible explanation for this is that the images are dominated by the relatively slow, respiratory frequency signal; if the adult images were filtered to highlight perfusion effects differences between the corrected and uncorrected images may become apparent.

Using a linear function to determine the delay on each electrode combination is sensible for this system, is it has been confirmed by Carefusion ( http://www.carefusion.com ) that the extra delay when the drive combination is switched, every 13 electrode combinations, is trivial compared to when only the sense electrode combination is changed, as is the pause at the end of the data frame. It was initially thought that such pauses could be the cause of the non-linear component of correlation in the corrected data (Fig.4), but it now appears unlikely. 
It should be noted that, at least in theory, the data collection order could be altered such that reciprocal measurements were consecutive, resulting in a much lower standard deviation of the reciprocity errors. This however would not improve image accuracy, and could actually make it worse since the temporal averaging, provided by reciprocal pairs, has been removed.

Often sets of images are averaged to reduce noise related artefacts, whilst this has not been tested in this paper it should be noted that the errors are not spatially uniform and it cannot be assumed that errors will average out. Errors are lower where changes slower, e.g. peak inspiration and expiration, but these points are not always the focus of a study, and as previously noted, the peak may be detected a data frame later in some electrode combinations than others, even within one reciprocity pair.

More work is needed to determine how fast a frame rate is needed, relative to frequencies of the physiological signals, for the lag effect to become insignificant. This could include looking for variation in derived parameters, such as those proposed by Gómez-laberge et al (2012). However a conservative estimate is that 65 frames per cycle is sufficient $(13 \mathrm{~Hz}$ frame rate / adult breathing rate $0.2 \mathrm{~Hz}$ ), but 15 frames per cycle is insufficient $(13 \mathrm{~Hz}$ frame rate / neonate breathing rate $0.91 \mathrm{~Hz})$. Ideally a frame rate should be chosen to reduce lag errors to less than the quantisation error of the system. For a system with 12 bit analogue to digital conversion, with the gain adjusted so the maximum boundary voltage in the baseline frame is $80 \%$ of the dynamic range, maximum tidal breathing changes are of the order of $1 \%$ of the dynamic range. Approximating breathing as a sine wave, the frame-rate required to limit the maximum change between frames to the quantisation error $\left(1 / 2^{\wedge} 12\right)$ is 52 frames per cycle. It should be noted that requirement applies to the physiological signal of interest and any other physiological signal which may be large enough to affect the reconstruction. Thus if only the tidal breathing respiratory signal is to be considered, and the cardiac frequency signal is negligible compared to this, a slower frame rate may be acceptable than for a study of blood perfusion, where the images will be filtered to highlight the small cardiac signal. Where signals that are not period sinusoids, the highest frequency component of interest needs to be considered.

Thus the required frame will vary widely depending on the age of the subject and the signals of interest. Where it is not practical to achieve a sufficiently fast frame rate, and for retrospective analysis of previous data, lag correction is a viable alternative - as long as the EIT system used is well characterised. Some EIT systems, like the one used here, have a single current source and measurement circuit, others, like the Sheffield Mark3.5 (Wilson et al 2001) are semi-parallel, applying current to each drive electrode combination in turn, but collecting from all the associated receive electrodes combinations simultaneously. Even in this semi-parallel set up, lags of up to F/(n2) will be present, where $n$ is the number of electrodes and $F$ the frame rate of the system, but the distribution of these lags will be totally different to that of a fully serial system. Thus lag correction needs to be tailored to each EIT system. With systems that inject multiple current frequencies simultaneously, such as the Sheffield Mark3.5, the same approach can be used, with lag correction applied individually to data from individual current frequencies.

In this study frequency domain lag correction was applied. An alternative, time domain, approach would be interpolation, however the relative merits of this, with respect to speed and accuracy is beyond the scope of this paper.

In conclusion, this lag effect cannot safely be ignored. Ideally EIT systems should be run at a frame rate which reduces lag to less than the quantisation error, i.e. for a 12 bit system, greater than 50 times the frequency of the dominant and interesting physiological signals. Where this is not practical, the intra-frame system timings should be determined and lag corrected for, as described in this paper. It is likely that both approaches will have their place, depending on system availability and the physiological signal of interest. 


\section{References}

Adler, A, 2004, Accounting for erroneous electrode data in electrical impedance tomography , Physiol. Meas. 25:1:227-238

Adler, A, JH Arnold, B Bayford, and A Borsic; Brown, B; Dixon, P; Faes, TJC; Frerichs, I; Gagnon, H; Garber, Y; Grychtol, B; Hahn, G; Lionheart, WRB; Malik, A; Patterson, RP; Stocks, J; Tizzard, A; Weiler, N and Wolf, GK, 2009, GREIT: a unified approach to 2D linear EIT., Physiol. Meas. 30:S35-S55

Adler, A; Amato, MB; Arnold, JH; Bayford, R; Bodenstein M, M; B"ohm, SH; Brown, BH; Frerichs, I; Stenqvist, O; Weiler, N and Wolf GK , 2012, Whither lung EIT: Where are we, where do we want to go and what do we need to get there?, Physiol. Meas. 33::679-694

Bayford, R; Kantartzis, P; Tizzard, A; Yerworth, R; Liatsis, P and Demosthenous, A, 2008, Development of a neonate lung reconstruction algorithm using a wavelet AMG and estimated boundary form, Physiol.Meas 29:6:S125-S138

Brown, BH and Seagar, AD, 1987, The Sheffield data collection system, Clinical physics and physiological measurement 8:Suppl A:91-97

G Hahn, A Just, J Dittmar and G Hellige, 2008, Systematic errors of EIT systems determined byeasily-scalable resistive phantoms, Physiological Measurement 29:S163-S172

Gómez-Laberge, C., Arnold, J.H., Wolf, G.K., 2012, A Unified Approach for EIT Imaging of Regional Overdistension and Atelectasis in Acute Lung Injury, IEEE TRANSACTIONS ON MEDICAL IMAGING 31:3:834-842

Grychtol, B, G K Wolf, A Adler, and J H Arnold, 2010, Towards lung EIT image segmentation: automatic, Physiol. Meas. 31:3:S31-S43

Grychtol, B; Lionheart, WRB; Bodenstein, M; Wolf, GK and Adler, A, 2012, Impact of Model Shape Mismatch on Reconstruction Quality in Electrical Impedance Tomography, IEEE transactions on medical imaging 31:9:1754-60

Hartinger, AE; Guardo, R; , 2009, Real-Time Management of Faulty Electrodesin Electrical Impedance Tomography, IEEE TRANSACTIONS ON BIOMEDICAL ENGINEERING 56:2:369377

Hartinger, AE.; Gagnon, H; Guardo, R, 2007, Accounting for hardware imperfections in EIT imagereconstruction algorithms, Physiol. Meas. 28:7:S13-S27

Minhas, AS and Reddy, MR, 2005, Neural network based approach for anomaly detection in the lungs region by electrical impedance tomography, Physiol. Meas. 26:4:489-502

Oh, TI; Woo, EJ and Holder D, 2007, Multi-frequency EIT system with radially symmetricarchitecture: KHU Mark1, Physiol. Meas. 28:S183-196

Tidswell, T; Gibson, A; Bayford, RH and Holder DS, 2001, Three-Dimensional Electrical Impedance Tomographyof Human Brain Activity, NeuroImage 13:283-294

Wilson A J, Milnes P, Waterworth A R, Smallwood R H and Brown B H, 2001, Mk3.5: a modular, multi-frequency successor to the Mk3a EIS/EIT system, Physiol. Meas 22:49-54

Yerworth, RJ and Bayford, R, 2007, A new clinical data and image analysis tool for monitoring neonatal lung function., IFMBE Proceedings 13th International Conference on Electrical Bioimpedance/8th Conference on Electrical Impedance Tomography. Graz, Austria. 17:388-391

Yerworth, RJ and Bayford, R, 2011, Robustness of Greit reconstruction algorithm with respect to reciprocity errors in the data, Proceeding of the 12th International Conference in Electrical Impedance Tomography. University of Bath

Fig. 1: Flowchart of lag correction and analysis

Fig.2: images reconstructed from normalised simulated data, with changes occurring only in the 
lung regions (scale arbitrary units): a)baseline b)static1, c)static2, d)serial-collection, e)difference between staticl and serial-collection images $(d-b)$

Fig.3: Effect of lag correction on one pair of reciprocity measurements, widely separated in time

Fig.4: Correlation between Standard deviation of reciprocity errors and time lag between data collection, for Neonate data a) uncorrected data, b) lag corrected data

Fig.5: images reconstructed from the Neonate data, displayed using the same impedance change scale (arbitrary units) a) uncorrected b) lag corrected, c) difference (b-a)

Fig.6: a) mean and b) standard deviation of differences between original and lag-corrected images for neonate (arbitrary units)

Fig.7: images reconstructed from the Adult data, displayed using the same impedance change scale (arbitrary units) a) uncorrected b) lag corrected, c) difference (b-a)

Fig.8: a) mean and b) standard deviation of differences between original and lag-corrected images for Adult, (arbitrary units) 
